\title{
El valor económico: una presentación de sus medidas
}

\section{Economical Value: a Presentation of its Measurements}

\section{Isidro Hernández Rodríguez}

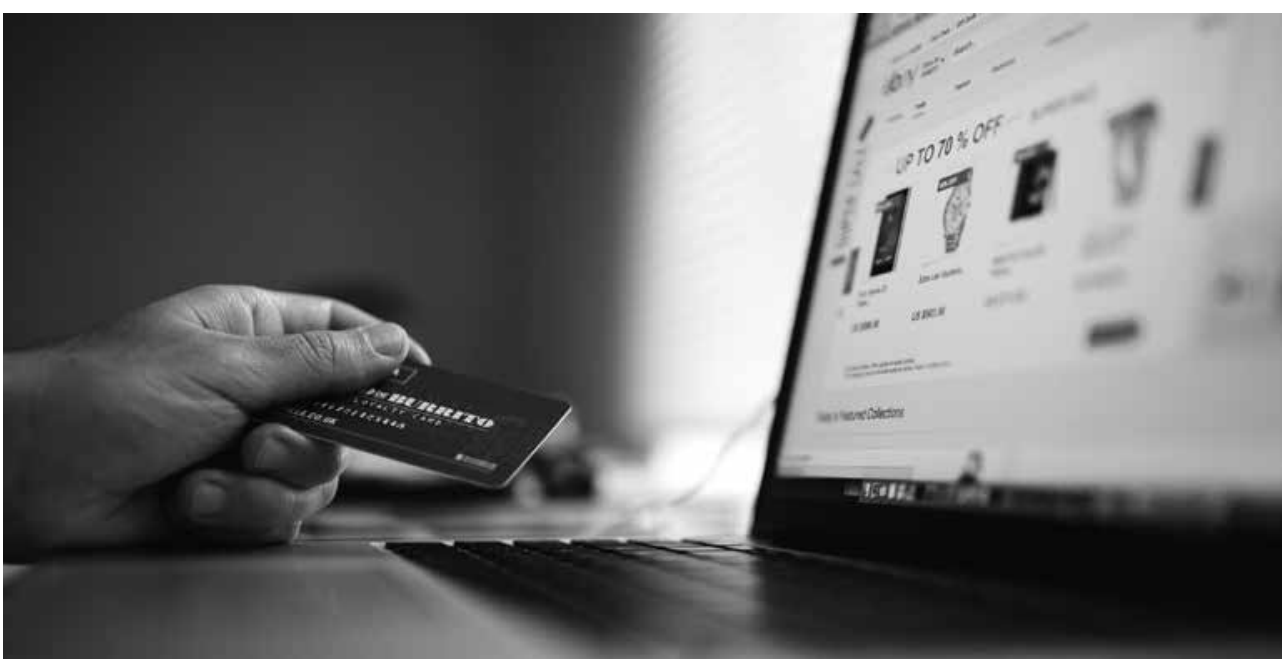

\section{Resumen}

¿Cómo valora el economista para poder entender que una cantidad de un bien es igual a una cantidad de otro bien o de dinero? ¿Cómo valora un conjunto de cosas heterogéneas para poder hacer simples operaciones aritméticas? Las respuestas a estas y otras preguntas similares remiten la atención a la teoría del valor, y este artículo se dedica a presentar, de manera didáctica, un único aspecto conceptual: la unidad de medida del valor. Cualquier profesional de la economía sabe que el tema del valor tiene una complejidad enorme porque está relacionado con la determinación de otros aspectos teóricos esenciales, tales como la teoría del capital, la teoría de la producción y la teoría de distribución. Este documento no trata estos aspectos, aunque tenga que aludir a ellos tangencialmente; su objetivo es exponer de manera sucinta y entendible el porqué de la necesidad de una unidad de medida de valor y las dificultades que enfrentan las teorías económicas en su búsqueda, siempre teniendo en mente un público lector con leves bases en economía (el especialista debe recurrir a otros textos rigurosos que desarrollan este tema). Para lograr tal objetivo, el texto se redactó de

\section{Citar este artículo como: Hernández, I. (2020). El valor económico: una presentación de sus medidas. Revista Papeles, 11(22), 22-42.}

Fecha de recibido: septiembre 5 de 2019 • Fecha de aceptación: noviembre 1 de 2019

* Profesor de la Universidad Externado de Colombia y la Escuela Colombiana de Ingeniería. Correo electrónico: isidro.hernandez@uexternado.edu.co 
forma progresiva: de lo más antiguo a lo más nuevo del pensamiento económico, del caso más sencillo al más complejo. Por ello, la primera sección se dedica a mostrar el origen de la necesidad de la medición del valor. Luego se pasa a mostrar por qué la teoría del valor es importante (al igual que la relevancia de la medición), lo que abre paso a exponer cuáles son los requisitos que debe cumplir una métrica del valor y las soluciones que han sugerido las diferentes escuelas de pensamiento económico, y se cierra con una conclusión.

Palabras clave: teoría del valor, medida del valor, historia de la economía.

\section{Abstract}

How do economists value anything in order to understand that certain amount of goods worth the same as another amount of goods, or money? How do they value a group of heterogeneous things in order to make simple arithmetic operations? The answers to these and some similar questions lead us to the theory of value, and this paper aims to present, in a didactical way, a single conceptual aspect: the unity of the value measurement. Any economic scholar knows that value has a huge complexity because it is related to some other essential theorical aspects, such as the theory of capital, the theory of production, and the theory of distribution. This document does not deal with such topics, although it has to mention them superficially. Its purpose is to briefly present why a unity of the value measurement is needed, and the difficulties faced by the economic theories in their searches, always taking in mind a reader with few bases in economics (specialists should turn to other books that deal with that topic). In order to achieve its purpose, the text was written in a progressive way: from classical to contemporary economical thinking, from simple cases to complex ones. Therefore, the first part of the paper deals with the origin of the necessity of value measurements. Then it shows why the theory of value is important (such as the importance of its measurement), what leads us to explain the requirements that a value measurement must fulfil, and which sorts of solutions have been suggested along the time; finally, it closes with a general conclusion.

Keywords: theory of value, value measurement, economics history.

\section{Introducción}

Si a un vendedor se le pregunta cuánto vale una unidad del producto que oferta, con certeza contestará que una cantidad determinada de unidades monetarias. En su respuesta una unidad de bien es equivalente a una cantidad de dinero. Si se le pregunta a un trabajador por el valor de su trabajo su respuesta es que tanto dinero por mes. Para él también un tiempo de trabajo, un mes, por ejemplo, equivale a una cantidad de dinero. Pero si se les pregunta al vendedor y al trabajador por qué esa cantidad de dinero y no otra, las respuestas empiezan a ser variadas, posiblemente algunas serán perplejas. $Y$ si se les indagara sobre lo que luego obtienen con el dinero recibido, manifestarían, el primero, que una cantidad de mercancías más una ganancia y, el segundo, una cantidad de bienes que integran su canasta de consumo.

En la vida práctica individual las respuestas estarán siempre referidas al valor monetario de los bienes, servicios, capital, tierra y trabajo. 
Cuando se pasa al ámbito social las preguntas y las respuestas se complican; y la persona estándar no entiende la mayoría de las circunstancias económicas que la envuelven. A la vista de los individuos es normal que todo esté valorado en moneda, pero cuando se pasa a la perspectiva de los economistas la moneda corriente desaparece. Todo se vuelve real.
No se habla de dinero sino de intercambio de bienes y servicios, de bienes de capital, de salario real, de tasa de interés real, etc. Lo mejor es que se hacen operaciones aritméticas con lo real. Por ejemplo, al ingreso real se le resta el consumo real y se obtiene el ahorro real; o se habla del capital agregado, es decir, de una suma de un conjunto de bienes.

\section{Origen del problema}

La palabra 'valor' tal vez ha desempeñado un papel demasiado importante en nuestra literatura. No hay un 'valor', sino que hay valores. No existe una cosa constante, permanente, aislable llamada 'valor', existen únicamente razones en que los bienes se cambian en circunstancias particulares de un día en particular y una hora también particular en el mercado (...) El término valor sugiere algo intrínseco a objetos o acciones físicas, que subsisten por derecho propio, que pueden ser almacenados en recipientes de seguridad garantizada, y vertidos desde uno de tales recipientes en otro. En cambio, el valor es, en cada caso momentáneo y especial, hijo de las circunstancias (Shackle, 1972; pp. 134-135).

En A Treatise of Taxes y Contributions, Petty (1899), como lo manifiesta en el subtítulo, busca establecer la naturaleza y la medida de un conjunto de variables. En el capítulo cuarto planteó el problema de la medición de la producción y la generación del ingreso o renta a partir de tomar un productor de maíz ${ }^{1}$, sin familia, que obtiene una cosecha de la cual descuenta la semilla que utilizó, la cantidad

1 En economía tradicionalmente se traduce corn como cereal, dado el problema que aquí se trata, se hace la traducción por maíz porque en el argumento de Petty el sentido es de un bien, no de una clase de bienes, la clase cereal. de maíz que usará para su autoconsumo, la parte que empleó para obtener telas y otros artículos necesarios (los otros bienes de su cesta de consumo), o maíz para intercambiar por otros bienes. Supone que el maíz que queda después de restar los gastos equivale a veinte bushels ${ }^{2}$, cantidad que denominó renta natural de un año. Luego supuso que el agricultor siembra durante varios años, o como dijo, "tantos años como el ciclo" de escasez y abundancia, y al promedio anual lo llamó "la renta ordinaria de la tierra en maíz", una especie de renta media de largo plazo.

Como la renta está representada en maíz, se preguntó sobre cuánto dinero vale esa renta. Para resolver la pregunta supuso que otro individuo trabaja en una mina y extrae plata que la refina y al final obtiene un peso de veinte onzas de plata. Luego la lleva al mismo lugar donde se encuentra el hombre que cosecha maíz -evita el costo de transporte-, y trabaja en la acuñación de monedas; es decir, el mineral de plata que obtuvo lo vuelve dinero -soslaya el costo de señoreaje-; por tanto, obtiene dinero equivalente a veinte onzas. En este proceso el minero-emisor también siembra y cosecha maíz para su sustento $\mathrm{y}$ hace los mismos descuentos que el primer agricultor.

2 Medida de capacidad de mercancías sólidas en los países anglosajones. Si es trigo es equivalente a 27,216 kilos, pero si es maíz desgranado es igual a 25,402 kilos. 
Petty implícitamente supone que el minero-emisor-agricultor obtiene el mismo nivel de cosecha que el agricultor, veinte bushels, e incurre en iguales gastos. El agricultor y minero-emisor-agricultor se encuentran en idéntica situación. Cuando llega a este punto "resuelve" su pregunta: la relación es veinte a veinte, por tanto, un bushels de maíz es igual a una onza de plata. Así obtuvo el precio del maíz. Acto seguido, a esa igualdad le da el nombre de equilibrio de valores; al tiempo que acepta que en el mundo práctico hay variedad y complejidad de intercambios porque existe una heterogeneidad de bienes. Reconoce que en las diferentes actividades los trabajos difieren; trabajar la plata puede requerir más arte y explotar las minas es más peligroso, y estos aspectos diferenciales hacen que se modifiquen las proporciones entre bienes, $y$ entre bienes y metales.

Utiliza como bien equivalente la plata, pero reconoce que en su época las cosas se miden con el oro y la plata, siendo el mineral de uso común la plata. Ante ese bimetalismo se pregunta por qué no hay dos medidas. $\mathrm{Su}$ respuesta es que entre muchas medidas la sociedad escoge la mejor. El problema práctico es determinar el peso y la finura del metal al momento de la transacción, pues son características que son difíciles de medir. Entonces supone una finura y un peso dados y observa que el precio de la plata varía porque cambian las proporciones con los otros bienes que mide, y el espacio y el tiempo juegan en la transacción: la geografía y la distancia a las minas modifican el precio, y la comparación del presente con el futuro hace cambiar la valoración.

Este procedimiento analítico del siglo XVII -anterior al nacimiento de la ciencia económica- para definir el concepto de renta natural permite identificar algunas cuestiones centrales de la teoría económica en el siglo XXI, entre ellas la que se aborda en este artículo: ¿cómo se miden las variables económicas, en específico el valor?

\section{Importancia de la teoría del valor}

$\mathrm{Al}$ definir la renta natural Petty tomó un monto global y restó cantidades, como se describió en la sección anterior. Por principio aritmético la operación de resta se hace entre elementos homogéneos, por eso se indicó que para cubrir los gastos se entregó parte del bien producido; es decir, la transacción se hizo en maíz, luego implícitamente se midió la tela en maíz, lo que le permitió restar al total producido de maíz una magnitud en la misma unidad de medida, así cumplió con el principio matemático.

Si se excluye el consumo de maíz del agricultor, los demás gastos implicaron un intercambio, maíz por otros bienes. Al hacer este razonamiento se introdujo otro problema, comparar bienes diferentes en unidades diferentes. Se necesita evaluar cuánta cantidad de un bien se debe entregar por otro para

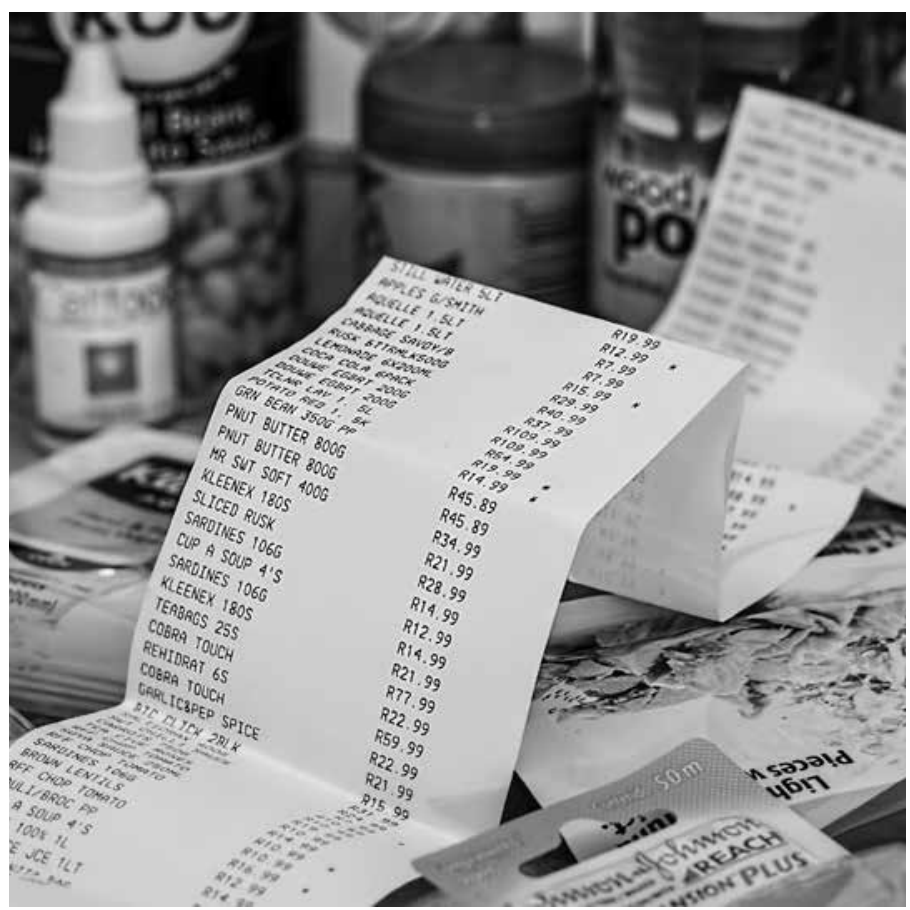


conseguir lo que llamó el equilibrio de valores. Esa valoración permite a su vez estimar si es o no costoso el gasto. No se trata de cualquier proporción matemática, por ejemplo, de un kilo de maíz por un metro de tela. Y no es arbitraria porque en esa razón se deben considerar variables cualitativas: ¿qué tipo de maíz y tela? Y para cerrar, Petty introdujo dinero, es decir, involucró el concepto de precio. La forma en que lo hizo llevó a una igualdad entre el valor y el precio de los bienes en consideración, tanta plata por tanto de maíz, luego una cantidad de plata por una cantidad de tela.

El proceso de Petty para definir una variable deja como enseñanza que un conjunto de operaciones y de relaciones dependen de la medición del valor. La teoría del valor es base y columna vertebral de la teoría económica. Sobre ella se sostiene y ella articula el conjunto de axiomas y postulados que permiten obtener corolarios significativos para realizar predicciones sobre del mundo concreto (Dobb, 1945). Los axiomas y postulados tienen que ser pertinentes con los hechos económicos, y la relación entre las variables se tiene que especificar de manera precisa y en correspondencia con el problema económico que se quiere estudiar. Tanto en el ámbito microeconómico, en el sectorial y en el macroeconómico, las relaciones entre las variables involucran la especificación de ecuaciones de definición, ecuaciones de comportamiento y funciones, de manera que los problemas se plantean en términos de un sistema de ecuaciones.

La teoría del valor es base y columna vertebral de la teoría económica. Sobre ella se sostiene y ella articula el conjunto de axiomas y postulados que permiten obtener corolarios significativos para realizar predicciones sobre del mundo concreto
Tómese el caso del maíz que plantea Petty. Si hay una oferta de maíz de una calidad específica por parte de un agricultor, la pregunta teórica es: ¿de qué depende la oferta de maíz? Y esta cuestión indica que se tiene que concebir una hipótesis que se traduce en una función: la cantidad ofertada se relaciona de una manera específica con un conjunto de variables que reciben el nombre de argumentos. Si dentro del conjunto de argumentos se involucra el precio de mercado del maíz se tiene que plantear otra hipótesis sobre la formación de ese precio, el cual se plasma a su vez en otra ecuación. Si ese precio se parametriza, esto es, se convierte en una constante, se evita la nueva función; pero debe existir una razón robusta y conectada con los hechos reales que justifique la parametrización. Una forma clásica de hacerlo es que el precio lo fije el mercado, lo cual lleva a la necesidad de plantear una función de demanda que también tiene como argumento el precio. Como la solución del problema requiere del precio de mercado, se postula la igualdad entre la oferta $(S)$ y la demanda de maíz $(D)$, lo que introduce una ecuación adicional o ecuación de cierre. El desarrollo de este caso sencillo de tipo micro conduce a especificar un sistema de tres ecuaciones: dos funciones y una ecuación de cierre.

Se puede elevar el problema al ámbito sectorial. El interés ya no está en la oferta de un productor sino en la de varios empresarios del sector agrícola, y no en un consumidor, sino en el conjunto de demandantes de diversas calidades de maíz. Y si se preguntara, a manera de ilustración, por qué se oferta maíz y no otro bien o servicio, de dónde obtienen los ingresos los compradores y por qué ese nivel de ingreso de los demandantes, entonces, el problema subiría al ámbito macroeconómico. En el agregado ya no es válida la lógica de lo micro, de los agentes, del productor y el consumidor, pues su actuación y toma de decisiones quedan limitados por lo agregado; el entorno social toma un movimiento autónomo: primero tendría que indagarse por 
los argumentos del nivel de utilización de los factores productivos que lleva a la obtención de un nivel de producto global, producto que tiene al menos dos componentes sectoriales, el producto de bienes de capital y el producto de bienes y servicios de consumo. Llegados a este punto se analizan los determinantes de la distribución entre todos los oferentes de factores, y dada esa distribución del ingreso, ahora sí indagar sobre el ingreso individual, el ingreso que se convierte en la restricción presupuestal que afectará la demanda individual de un conjunto de bienes y servicios de capital o de consumo final, por ejemplo, el maíz que se requiere como insumo intermedio o para su consumo final. En pocas palabras, este análisis está en el nivel agregado; la restricción presupuestal de un agente microeconómico, un consumidor, depende de la forma como se distribuye el producto social en un momento determinado. Las fuerzas globales determinan las variables sectoriales y microeconómicas. No es lo individual lo que determina lo social, sino lo social lo que determina la dinámica individual.

En este ejemplo la cantidad de maíz puede estar medida en unidades de peso, por ejemplo, en kilos. Al poner los argumentos de las funciones se introducen variables económicas que requieren medida específica. El precio implica el valor; al subir de nivel el problema se involucró el ingreso, y detrás del ingreso está el valor; y cuando se llega al nivel macroeconómico se tienen las variables agregadas, esto es, la suma de las unidades en que está medida la variable, por ejemplo, el ingreso agregado es la suma del ingreso al trabajo, al capital y a la tierra; por tanto, para poder sumarlas deben ser homogéneas. ¿Qué hace homogéneo los ingresos de esos tres factores? La respuesta es la valoración, y valorarlos significa aplicarles una medida, y esa medida se llama valor. Obsérvese que esto implica teóricamente tener una medida homogénea de valor en su máxima manifestación porque el valor permite homogenizar variables inicialmente representadas en cantidades medidas de forma heterogénea $\mathrm{y}$, al sumarlas, obtener el agregado.

Así las cosas, la importancia de la teoría del valor radica en que permite relacionar de manera lógica e integral el conjunto de ecuaciones que constituyen la teoría económica, y en que especifica la medida de las variables económicas. Como de manera sintética lo manifiesta Dobb (1945), su importancia está en que le provee a la teoría económica "el sistema de principios generales presentados en forma cuantitativa" (p. 11).

\section{Las condiciones de la medida del valor}

But within the field of mathematics the problem of quantitative definitions of concepts is not a problem separate from what we do with the concept. Indeed, the concepts of mathematics obtain their quantitative meaning through the logical operations we perform on them. In pure mathematics there is no such problem as quantitative definition of a concept per se, without reference to certain operations. Therefore, when economists talk about the problem of quantitative definitions of concepts and laws in economics, they must have in mind something which has to do with real phenomena (Haavelmo, 1941, p. 4).

En ciencia la precisión de los conceptos evita indeterminaciones, múltiples significados. Cada término que se incorpore en el acervo de conocimiento debe ser delimitado, de tal manera que capte las propiedades esenciales del objeto o unidad de estudio. Si la variable es de tipo cuantitativo, el significado de la medida debe ser exacto. Si el objeto de estudio plantea relaciones entre variables cuantitativas, la teoría relaciona cantidades cuya unidad de medida debe ser precisa. 
En economía es importante distinguir dos aspectos conceptuales. El primero es la diferencia entre la medida de una variable teórica y la medida de la variable en la actividad empírica. La medida empírica trata de acercarse, con algún grado de error, a la medida teórica. Una cosa es dinero en teoría y otra cosa son las mediciones empíricas del dinero. ¿Cuánto se acerca la una a la otra? ¿Cuál es el grado de error aceptado por la comunidad de investigadores? El segundo aspecto corresponde a la medición de la relación o de la dependencia teórica entre variables. Cuando se pasa a la instancia empírica el cálculo del planteamiento teórico se hace con instrumentos estadísticos, previa la identificación de si en la base teórica se plantea una relación o una dependencia, pues eso determina el método de medida ${ }^{3}$. Si en teoría se plantea una relación entre variables, en la práctica se pueden usar técnicas estadísticas de estimación de coeficientes de correlación, pero si en teoría se plantea una dependencia entre variables, en la práctica ya no es consistente el uso de técnicas de correlación; se tienen que utilizar otros métodos estadísticos, por ejemplo, las cópulas. Adviértase que el planteamiento teórico determina el método práctico de medición, los métodos estadísticos no se pueden utilizar de manera arbitraria.

El cálculo de la relación o de la dependencia no puede evitar el primero de esos aspectos. Para hacer la estimación ya deben estar calculadas las variables. Si la teoría asume en su explicación una variable cualitativa, categórica o no métrica, es necesario "medirla", para lo cual se lleva a una escala de medición. La escala nominal se utiliza para la relación de identidad entre un número y el objeto de medida (cero si es hombre y uno si es mujer), y la escala ordinal para mantener el orden de la característica (la preferencia y la indiferencia),

3 En estadística la dependencia implica unas exigencias estadísticas más rigurosas que los requerimientos de la correlación. sin que en esas escalas el número asociado tenga un significado, y menos el teórico. En el caso del género cero no significa cantidad nula porque la variable no es métrica.

En ciencias sociales, en especial en economía, se argumenta que sus variables se pueden medir con base en proposiciones alternativas a la medición clásica, asociadas a la física (Boumans, 2015). Esto significa que medir no implica métrica, que no es necesaria la unidad de medida, ya que se propone un procedimiento en el que se tiene un valor de referencia para un objeto que se asume como conocido, que experimentalmente se compara con valores de otros objetos y con otros valores del objeto, el cual se mide de tal manera que al final se asigna $e l$ valor (Mari, 2007). En el caso de la teoría axiomática de la representación, bastaría con cumplir los principios de las asignaciones numéricas para tener la medida, el número asignado es en sí mismo la unidad (Boumans, 2007 y Michell, 2007). En varias de las propuestas se considera que calcular un coeficiente de correlación hace parte del proceso de medición; en este caso, ¿cómo se miden las variables involucradas para obtener el coeficiente si una de ellas es la que se "mide"?

Todas las teorías económicas se construyen con variables que tienen métrica. El número asociado a la variable implica una interpretación unívoca con respecto al objeto de medida. $\mathrm{Y}$ esto es más relevante al pasar al momento empírico, pues:

Cuando compramos algo en una tienda y el vendedor dice 'tres chelines, por favor', su 'tres' no es un mero símbolo matemático que signifique 'el tercer término después del comienzo de alguna serie'; su 'tres', en realidad, no puede ser definido por sus propiedades aritméticas (Russell, 1923, p. 247).

Así las cosas, y de acuerdo con los dos aspectos conceptuales señalados al principio y con el epígrafe de la sección, el valor, la piedra angular de la teoría económica, es una variable 
métrica, en consecuencia, su medición se tiene que hacer en el sentido clásico, de laboratorio. Esa medida debe cumplir con los siguientes parámetros:

i. Es una magnitud que recoge la propiedad esencial del valor.

ii. La magnitud puede ser un escalar o un vector.

iii. Tiene una unidad que se adopta como estándar o norma, por tanto, es:

a. Una magnitud que capta la cantidad del mismo tipo del mensurando y que sirve para tomarse como estándar, como unidad.

b. Invariante: es una constante universal que tiene múltiplos y submúltiplos.

c. Reproducible: una persona la pude reproducir en cualquier momento $\mathrm{y}$ espacio y debe llegar al mismo resultado.

iv. Admite las operaciones de adición y sustracción.

v. La propiedad de invariante permite identificar de manera precisa la magnitud del cambio de la variable a lo largo del tiempo.

\section{Las propuestas de medida del valor}

The Method I take to do this, is not yet very usual; for instead of using only comparative and superlative Words, and intellectual Arguments, I have taken the course (as a Specimen of the Political Arithmetic? I have long aimed at) to express myself in Terms of Number, Weight, or Measure; to use only Arguments of Sense, and to consider only such Causes, as have visible Foundations in Nature (Petty, 1899, p. 207).

Desde el siglo XVII se proponen medidas de valor y los economistas tienen un conocimiento acumulado, que se logró gracias a la crítica, de qué no debe ser el valor y qué relaciones tiene con otras variables del sistema de ecuaciones. Uno de los que dio el primer paso fue Petty. Consideró que, para evitar opiniones generales de uso corriente sobre la situación económica del Reino Unido de su época, se tienen que calcular las variaciones de los hechos económicos con medidas físicas, con "estándares y medidas naturales" (Petty, 1899, p. 77). Propone que los bienes deben ser valorados por la tierra y el trabajo, que considera son denominaciones naturales, pues todos son producto "de la tierra y las labores de los hombres. La tierra y los hombres se pueden expresar "en términos de número, peso o medida”, como sentencia el epígrafe

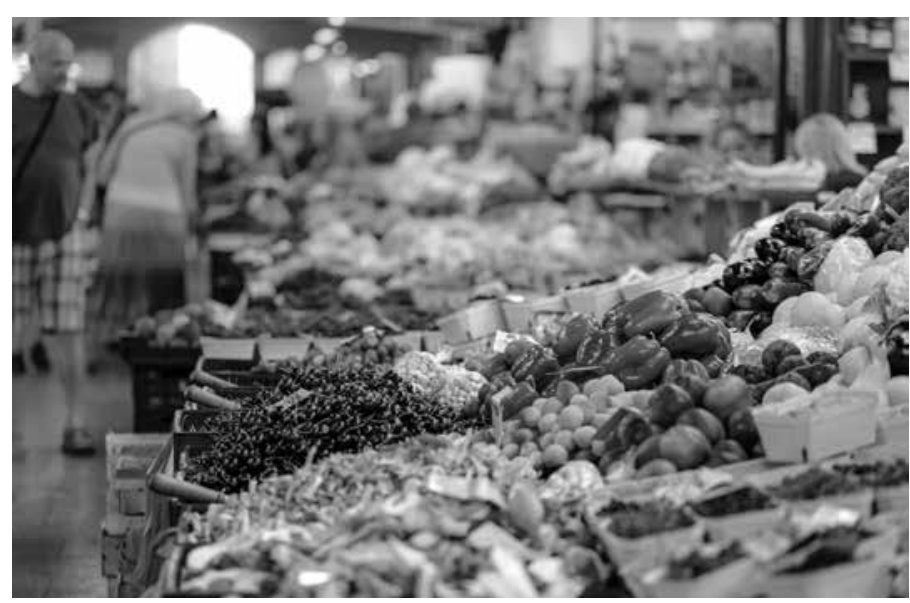

que encabeza la sección. La tierra tiene una medida de longitud, pero no especificó la medida de las labores de los hombres. En pocas palabras, no encontró un común denominador de la heterogeneidad de tierras y de trabajo y el común denominador de la tierra y el trabajo o el valor propio, matemáticamente hablando, que permita la conversión.

En el caso de su definición de renta natural en la que utiliza el intercambio de maíz por tela o por plata, no identificó las propiedades de la relación de intercambio que le permitieran plantear un concepto de valor económico. Se quedó al nivel de Aristóteles, reconocer que en el intercambio se relacionan dos valores, que los bienes tienen valor, pero que no se precisa 
su unidad de medida. Inclusive, la forma en que enunció la variable renta ya involucra dos problemas: la definición de la renta propiamente dicha, que está ligada a la medida de valor, y la delimitación del calificativo natural, concepto de uso común en el siglo XXI en la economía, por ejemplo, tasa natural de desempleo. ¿Qué significa natural en teoría económica? ¿Es un término impreciso que depende del contexto en que se usa?

Petty planteó la idea de que una unidad de medida física para determinar el valor debe tener la propiedad de ser reducida a otra por medio de un parámetro de conversión: los valores de la tierra homogénea, reducirlos al trabajo uniforme, y ambos reducirlos a los bienes. Casi un siglo después Richard Cantillon retomó la idea y sugirió el concepto de valor intrínseco, que utilizó como sinónimo del concepto precio. Ese valor tiene como unidad básica de medida la tierra y el trabajo que se utiliza en la producción. Reconoce que la producción agropecuaria es diferente por acre de tierra - usa el trigo y las ovejas como productos-. Para llegar a la medida supone homogeneidad en la calidad de la tierra y en la calidad del trabajo, y concluye que el valor de las producciones de dos parcelas diferentes debe ser el mismo. Toma la lana de una parcela y la utiliza como insumo en la producción de vestidos de estameña y la lana de la otra parcela es insumo en trajes de paño fino. En la producción manufacturera toma trabajos de calidades heterogéneas y cantidades de obreros diferentes en períodos de producción diferentes:

\section{Los bienes deben ser valorados por} la tierra y el trabajo, que considera son denominaciones naturales, pues todos son producto "de la tierra y las labores de los hombres. La tierra y los hombres se pueden expresar "en términos de número, peso o medida".
Una libra de lino convertida en finos encajes de Bruselas, exige el trabajo de catorce personas durante un año, o el de una persona durante catorce años, el precio pagado por esos encajes basta para pagar el sustento de una persona durante catorce años, y pagar, por añadidura, los beneficios de los empresarios (Cantillon, 1950, pp. 27-28).

La cantidad de trabajadores utilizados en la producción de una unidad de producto alarga o acorta el período de producción. Y el valor del trabajo lo mide en términos de la cantidad de bienes que se le entrega al obrero. Detecta que esa cantidad varía por la diferencia de productividad de la tierra, pero ahí trunca el razonamiento y se limita a hacer la conversión de la cantidad de bienes producidos por acre, de manera que es la medida física de la tierra la que equivale al valor intrínseco de la producción que se llevará al intercambio. En la segunda parte de su ensayo introduce el concepto de dinero y lo utiliza como unidad de precio de mercado, pero bajo el principio del precio como:

medida común de los valores corresponda, en forma real e intrínseca, es decir, en el precio de la tierra y del trabajo, a las cosas que a cambio de ellas se reciben. De otro modo la moneda sólo tendría un valor imaginario (Cantillon, 1950, p. 76).

En lugar de ver en el período de producción y en el valor del trabajo, la cesta de bienes que recibe un obrero, un determinante de su valor, ve y convierte a los factores de producción en la medida: "el precio o valor intrínseco de una cosa es la medida de la cantidad de tierra y de trabajo que intervienen en su producción, teniendo en cuenta la fertilidad o producto de la tierra, y la calidad del trabajo" (Cantillon, 1950, p. 28). Las tierras se cuentan en acres y los trabajadores por unidad, bajo el supuesto de homogeneidad en productividad. Esto le permitió sostener que la riqueza de un país se mide por la cantidad de trabajadores, del nivel 
de empleo. Desde luego, si las productividades de los factores afectan el valor de la producción que se lleva al mercado, estos no pueden ser invariantes, y el valor no puede tomar como unidad de medida una medida de superficie como el acre ni la cantidad de obreros en la producción. Nuevamente hace falta el factor de conversión de diferentes tipos de trabajadores, su reducción a una unidad común.

Pese al yerro conceptual, con su argumento dio un paso adicional frente a Petty: consideró la utilización de obreros a los que se les paga un salario, enlazó producciones sectoriales y cantidades de mano de obra diferenciada y percibió que la medida del valor de la producción no es independiente de la calidad de los insumos, fertilidad de la tierra y productividad del trabajo. Ya dejó prevista la dificultad en la medida del valor.

La tradición de medida de la economía clásica empezó con Smith, quien especificó el problema: "Primero, cuál sea la medida de este valor en cambio, o en qué consiste el precio real de todos los bienes", y "Segundo, cuáles son las diferentes partes integrantes de que se compone este precio real" (Smith, 1981, p. 30 ). A la cuestión responde que en el cambio "El trabajo, por consiguiente, es la medida real del valor en cambio de toda clase de bienes" (Smith, 1981, p. 31). Concepto diferente al de cantidad de trabajadores. El trabajo es la pena y la fatiga de trabajar, lo que se compra con dinero se produce con trabajo: "El trabajo fue, pues, el precio primitivo, la moneda originaria que sirvió para pagar y comprar todas las cosas" (Smith, 1981, p. 31). El trabajo es la medida absoluta del valor o valor absoluto, que en algunos apartes propone que se mide por el tiempo de trabajo.

Ese valor no es la medida para estimar el valor que toma en concreto en el intercambio, el valor relativo; pues,

es difícil averiguar la relación proporcional que existe entre cantidades diferentes de trabajo. El tiempo que se gasta en dos diferentes clases de tarea no siempre determina de una manera exclusiva esa proporción. Han de tomarse en cuenta los grados de fatiga y de ingenio (Smith, 1981, p. 32).

Además, en el proceso de cambio entra el deseo de cada una de las partes en negociación por pagar menos, lo que denomina el regateo. El intercambio no es exacto, se paga un valor diferente, al valor de la producción. Se tienen entonces dos valores, el de producción y el de mercado; por tanto, se requieren dos medidas.

Cuando entra el mercado, diferente al intercambio de bienes, la relación se hace con dinero. Y el dinero de Smith es el patrón plata, dinero que implica extracción del mineral y, por tanto, valorado con trabajo más el señoraje. El trabajo para todos los bienes, incluida la plata, lo ejerce el trabajador, y por su labor se le paga un salario, una cantidad de bienes o cesta de consumo obrero. Para Smith esta canasta no valoriza el trabajo. Así que el trabajo es la medida del valor. Para determinarlo mira las dos partes que entran en la negociación. El vendedor de trabajo, el obrero, y su comprador, el empresario.

Visto desde el obrero, el trabajo se valora por la pérdida o sacrificio "de comodidad, libertad y de felicidad" (Smith, 1981, p. 32) que sufre el trabajador. Es lo que Smith llama "el valor para el trabajador” (Smith, 1981, p. 33). Supone que el valor del sacrificio es igual en cualquier tiempo y lugar, sobre él no influye la cesta de bienes siempre que se presuponga igual estado de salud, fuerza, temperamento, aptitud y destreza. Al suponer que el obrero valora su trabajo con el costo de oportunidad de esos tres elementos, sin entrar en consideración que no sacrificarlos implica perder la cesta que le garantiza su vida, y que en consecuencia debe valorarla al tomar la decisión de trabajar. Entonces, Smith genera una constante, parametriza, sin suficiente argumentación que le permita aislar o convertir en independiente la variable de medida de una de sus características determinantes. ¿Qué será más valorado por el obrero: la comodidad, libertad y la felicidad 
o los bienes mínimos para mantenerse vivo? ¿Cuál es el valor de la vida?

Con esta secuencia argumentativa entró en un atolladero porque a) uniforma las destrezas, la salud, etc., esto es, homogeniza de forma arbitraria las cualidades individuales que determinan las cantidades de trabajo de individuos heterogéneos; b) si no son uniformes las cualidades individuales, la secuencia lógica del argumento dirá que la cesta de bienes de cada tipo de trabajador determina el valor del sacrificio; o induce un argumento circular si no hay independencia entre el costo de oportunidad calculado por el sacrificio de comodidad, libertad y felicidad, y el costo de oportunidad medido por la cesta mínima para mantenerse vivo; y c) porque el valor del trabajo, el sacrificio, es una variable no métrica.

Visto desde el empleador, el valor cambia al entregar diferentes cantidades de bienes según las circunstancias de negociación con el trabajador en el mercado laboral, del regateo. Ese es el valor de mercado del trabajo, no del trabajador. El valor de los bienes que integran la cesta no cambia pues están valorados desde la perspectiva del trabajador con su sacrifico valorado con el costo de oportunidad.

La cuestión de la medida se complica cuando responde la segunda pregunta que se planteó, los componentes del valor. El valor de la producción es igual a la cantidad de trabajo utilizado, a la cuantía de la unidad de medida, pues el trabajador, el individuo, añade valor a

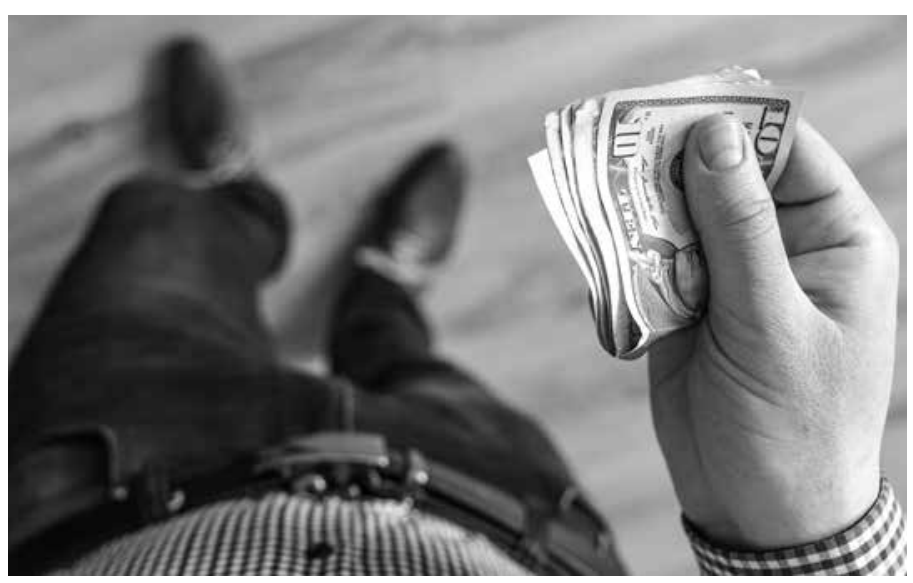

los insumos, agrega trabajo (Smith, 1981, p. 48). Ese valor agregado se distribuye entre los factores que se utilizaron en la producción, el trabajo, el capital y la tierra; esto es, entre el pago al trabajo, el salario o la cesta de bienes de los obreros, el pago al capital, o ganancia del empresario medida en trabajo, y el pago por el uso de la tierra o renta del suelo medida en trabajo. Es lo que se llama el costo de producción o precios de producción.

La suma o agregación de los tres valores, el valor distribuido, debe ser igual al valor total del producto. Pero en el valor del producto queda incorporado el valor de mercado del trabajo, la cesta de bienes del obrero valoradas desde el empresario, no del trabajador, no está medida en valor absoluto; para el empleador es un valor relativo, la cantidad de bienes que entrega con respecto a la cantidad de trabajo que recibe. Si la cesta de bienes de los obreros influye sobre el valor relativo del trabajo, nuevamente se cae en esa circularidad. Por esta vía, como todas las variables que integran el costo de producción están medidas en trabajo y existe una interdependencia entre la valoración del producto y la distribución del valor generado por el trabajo, la unidad de medida que propone no garantiza el principio de perfecta exogeneidad frente al objeto medido.

Para finalizar, supóngase que no existen razonamientos circulares: ¿cuál es la unidad de medida del trabajo, de las penas y pesares o del sacrificio? Las penas y pesares, así como el sacrificio, son variables no métricas. No hay unidad de medida, no hay una magnitud.

El aporte de Smith fue especificar con mayor precisión el problema, delimitación que le dio claridad y permitió que los teóricos que lo siguieron retomaran el problema de forma más limpia, más clara. Además, propuso un candidato a hacer el papel de valor absoluto, con una justificación robusta: el trabajo es el que agrega el valor, luego es en el trabajo donde se puede encontrar la medida. Justificación vigorosa, pero sin alcanzar el resultado, una medida de valor, una invariante. 
El planteamiento del sistema económico de Smith, de sus ecuaciones implícitas, se discutió durante el cuarto de siglo posterior a 1776, pero fue en la segunda década del siglo XIX cuando se plantearon alternativas teóricas robustas en torno a la teoría del valor y a la teoría de la distribución (Hollander, 1979).

David Ricardo sintetizó el problema en los siguientes términos:

Las únicas cualidades necesarias para que una medida del valor sea perfecta son: que ella misma tenga valor, y que ese valor sea invariable en sí mismo, del mismo modo que en una media perfecta de longitud la medida ha de tener longitud y esa longitud no debe estar expuesta a ser aumentada o disminuida; o en una medida de peso, que debe tener peso y ese peso debe ser constante (...) Aunque es fácil decir lo que debe ser una medida perfecta del valor, no es igualmente fácil encontrar una mercancía que tenga las cualidades requeridas (Ricardo, 1960, p. 275).

Ricardo no acepta el argumento de Smith sobre el valor absoluto. Dedicó el primer capítulo de los Principios a analizar las dificultades de la medida de valor a partir de desglosar el análisis de Smith. Si en la sociedad no hay compra-venta de trabajadores, todo el producto del trabajo, el valor producido, es de propiedad del trabajador; pero cuando aparece el mercado laboral, el valor absoluto del producto se reparte entre capital y trabajo, como lo plantea Smith, esas son las partes que componen el valor; así las cosas, la acumulación de capital afecta las ganancias; por tanto, modifica el valor absoluto del bien. En opinión de Ricardo, la composición entre ganancias y salarios no modifica el valor en cambio, el valor relativo de los bienes, pero la cantidad de trabajo requerido para producir un bien sí se modifica con la durabilidad del capital que se utiliza en la producción, lo cual conduce a que las composiciones entre capital y trabajo afecten el valor.
Ante la circularidad y el vacío argumentativo, abandonó la perspectiva de buscar un valor absoluto y concentró la cuestión en el valor relativo o valor de cambio, es decir, "el poder que tiene una mercancía de adquirir una cantidad dada de otra mercancía, sin referencia ninguna a su valor absoluto" (Ricardo, 1923, p. 301). Como para determinar el denominador se necesita un valor constante, un valor absoluto, el cual no existe, optó por asumir que

aunque reconozco plenamente que el dinero hecho con oro está sujeto a la mayor parte de las variaciones que sufren las demás cosas, lo supondré invariable, y, por ende, supondré también que todas las alteraciones en precio fueron ocasionadas por alguna alteración en el valor del bien del que puedo estar hablando (Ricardo, 1973, p. 34).

En estos términos, Ricardo muestra que no existe independencia entre la teoría del valor y la teoría de la distribución del producto, lo que impide determinar de manera precisa la medida del valor en términos de trabajo. A esto se suma el problema de la relación entre el valor absoluto, el precio de producción y el precio de mercado; este último calculado en términos de moneda metálica, cuyo valor es variable. La teoría económica quedó así cuasiindeterminada.

La siguiente proposición se debe a Marx. Reconoce, como Smith y Ricardo ${ }^{4}$, que cualquier mercancía tiene cualidad y cantidad y concentra su esfuerzo analítico en la característica de cantidad: "el valor de cambio se presenta como relación cuantitativa, proporción en que se intercambian valores de uso de una clase por valores de uso de otra clase, una relación que se modifica constantemente según el tiempo y lugar"5 (Marx, 2001, p. 45).

4 Meek (1956) hace un análisis detallado de las teorías del valor de Smith, Ricardo y Marx; y Sherman (1972) presenta el debate de la teoría del valor.

5 Todas las cursivas de las citas corresponden al original. 
"Es sólo la cantidad de trabajo socialmente necesario, pues, o el tiempo de trabajo socialmente necesario para la producción de un valor de uso, lo que determina su magnitud de valor"

El común denominador en la relación es "el trabajo abstractamente humano", "indiferenciado, esto es, de gasto de fuerza de trabajo humana sin consideración a la forma en que se gastó la misma" (Marx, 2001, p. 47).

En estos términos, la relación cuantitativa, el valor relativo, no es una constante y no es el trabajo, como lo argumentaron Smith y Ricardo, sino la fuerza de trabajo ${ }^{6}$.

Identifica el problema y propone una posible solución:

Un valor de uso o un bien, por ende, sólo tiene valor porque en él esta objetivado o materializado trabajo abstractamente humano. ¿Cómo medir, entonces la magnitud de su valor? Por la cantidad de 'sustancia generadora de valor' -por la cantidad de trabajo- contenida en ese valor de uso. La cantidad de trabajo misma se mide por su duración, y el tiempo de trabajo, a su vez, reconoce su patrón de medida en determinadas fracciones temporales, tales como la hora, día, etcétera (Marx, 2001, pp. 47-48).

La lógica de su argumento es que la cantidad de trabajo genera el valor, la que se mide en tiempo, luego la unidad de medida es la hora de trabajo. Como varios fabricantes de un mismo bien lo producen en tiempos diferentes porque existen discrepancias en productividad, entonces, estrecha su propuesta: "Es sólo

6 Cataño (2009) hace una presentación clara de toda teoría de Marx al alcance de un conjunto amplio de lectores. la cantidad de trabajo socialmente necesario, pues, o el tiempo de trabajo socialmente necesario para la producción de un valor de uso, lo que determina su magnitud de valor" (Marx, 2001, p. 48).

Enfrenta el problema de la heterogeneidad del trabajo tanto en preparación como en habilidad con una reducción a una unidad, el trabajo simple o menos calificado:

Por más que una mercancía sea el producto del trabajo más complejo su valor la equipara al producto del trabajo simple y, por consiguiente, no representa más que una determinada cantidad de trabajo simple. Las diversas proporciones en que los distintos tipos de trabajo son reducidos al trabajo simple como a su unidad de medida, se establecen a través de un proceso social (Marx, 2001, p. 55).

$\mathrm{Al}$ plantear una unidad de medida, casi en términos de un índice de trabajo, la dificultad a enfrentar es el cambio técnico y la composición entre capital variable y constante de cada empresa o sector económico. El escollo de la unidad de medida es el siguiente: las mejoras en maquinaria y equipo aumentan la cantidad de bien producida por unidad de tiempo, lo que lleva a dos escenarios. En el primero, la diferenciación de técnicas de producción entre empresas que elaboran un mismo bien. Esta diferencia la supera con el argumento que la producción con la técnica más eficiente reduce el valor, pues se disminuye el tiempo socialmente necesario, cae el promedio, lo que conduce a que la producción de la técnica menos eficiente tenga socialmente una reducción del valor y por la competencia es candidato a salir del mercado.

El segundo escenario es más complicado. Se conoce que en una misma cantidad de tiempo social con presencia de diferentes técnicas se producen diferentes cantidades de un mismo bien, entonces, el valor del bien se reduce. ¿Esto implica que se modifica la magnitud de valor absoluto? Su respuesta es que la 
magnitud de valor es constante si es constante el tiempo de trabajo socialmente necesario, pero este cambia o varía, luego "la magnitud del valor de una mercancía varía en razón directa a la cantidad de trabajo efectivizado en ella e inversa a la fuerza productiva de ese trabajo" (Marx, 2001, p. 50).

Como la fuerza productiva del trabajo varía, sostiene que

un cambio en la fuerza productiva del trabajo en nada afecta el trabajo representado en el valor. Como la fuerza productiva del trabajo es algo que corresponde a la forma útil adoptada concretamente por el trabajo, es natural que, no bien hacemos abstracción de dicha forma útil concreta, aquélla ya no puede ejercer influjo alguno sobre el trabajo. El mismo trabajo, pues, por más que cambie la fuerza productiva rinde siempre la misma magnitud de valor en los mismos espacios de tiempo. Pero en el mismo espacio de tiempo suministra valores de uso en diferentes cantidades: más, cuando aumenta la fuerza productiva, y menos cuando disminuye (Marx, 2001, p. 57).

Por consiguiente, la única forma para no alterar la magnitud del valor es que la productividad que surge del cambio técnico permanezca constante. Si bien su solución a las diferencias en las productividades con origen en la heterogeneidad de los trabajadores se soluciona con una unidad de trabajo simple, la dificultad que genera la heterogeneidad en la composición de capitales entre empresas de un mismo sector y los avances tecnológicos no quedó superada y la unidad no es invariante: el valor relativo surge de la comparación del tiempo promedio de los bienes y el valor absoluto del promedio del tiempo social. Como para calcular el relativo se necesita el absoluto, la tecnología cambia el tiempo promedio social, en consecuencia, intertemporal modifica la magnitud de valor.
Supóngase que Marx obtuvo la unidad de medida del valor: ¿soluciona los problemas del precio de producción y su relación con la distribución y de su correspondencia con el precio de mercado? La respuesta es negativa y esto derivó en el llamado problema de la transformación de valores a precio, que sigue aún vigente (Dostaler, 1978; Cuevas 1986; Hilferding et. al., 1974; Steedman, 1977; Tsoulfidis y Tsaliki, 2019; Wright, 2019).

Con Marx se cierra la propuesta teórica de establecer un sistema de ecuaciones, por tanto de magnitudes, que explica la dinámica de la economía capitalista con base en un conjunto de postulados sobre la producción de bienes, la distribución del producto entre quienes participan de su elaboración, la formación de los precios de los bienes, el nivel de precio y el valor del dinero con fundamento en una teoría del valor objetiva, objetividad que surge de colocar como centro el trabajo como único factor productivo capaz de crear valor y de tener valor en sí mismo. Al fracasar en la creación de una unidad de medida del valor el núcleo de la teoría no puede soportar la consistencia lógica que requieren magnitudes que dan cuenta de todo el sistema y se diluyen las conclusiones teóricas.

En los últimos veinticinco años del siglo XIX se consolidó una teoría que le dio un giro de 180 grados al análisis económico. Un conjunto de pensadores, entre los que se encuentran Jevons, Pareto, Menger, Von Wieser, Wicksell, Walras, Bates Clark, Fisher, Edgeworth y Marshall, propusieron un marco teórico que se cimienta en la teoría del valor subjetivo $^{7}$, y cuyos argumentos se fortalecieron en las tres primeras décadas del siglo XX con los aportes de Wicksteed, Pigou, Robertson, Von Hayek, Von Mises, Robbins, Cassel y Knight, y que a su madurez contribuyeron

7 MacVane (1893) muestra aspectos que se le discutían a la nueva teoría a partir de la teoría clásica, entendida como los planteamientos de Smith y Ricardo. 
Hicks, Allen, Arrow, Debreu, Samuelson, Hahn, Stigler, entre otros ${ }^{8}$.

Quien valora es el individuo al tomar sus decisiones de consumo de acuerdo con la felicidad que le proporcionan los bienes y servicios que prefiere; "El individuo es el único juez de lo que le gusta o disgusta" (Pareto, 1945, p. 51). La valoración la hace en términos egoísta, hedónico y racional, pues es un individuo que persigue la mayor felicidad para sí, de manera que en la selección de los bienes busca el placer y rechaza el dolor, y de forma consciente utiliza los medios disponibles para alcanzar el fin de máxima felicidad. El individuo tiene a su disposición un conjunto de cestas de bienes y servicios y elige una combinación de ellos, una cesta, que evalúa como la preferida comparada con respecto al resto del conjunto de cestas alternativas, es utilitarista. En la elección tiene la capacidad de ordenar sus preferencias, por tanto, puede ordenar de lo más preferido a lo menos preferido; y en la ordenación de preferencias sigue sus principios o juicios de valor, esto es, apela a su hedonismo reflexivo y a su propio interés, es egoísta. En síntesis, el individuo es el mejor juez de su propia felicidad, de su utilidad.

Cada combinación de bienes y servicios está asociada a un máximo de utilidad o felicidad y cuando hace efectiva su elección el individuo revela sus preferencias, está dispuesto a pagar un precio por la cesta preferida. Para poder pagar debe tener un ingreso, su restricción presupuestal. Obtiene el ingreso a partir de ofertar los factores productivos que posee. De los tres factores posibles -tierra, trabajo y capital-, al menos tiene uno que ofrecer al mercado. La teoría no se pregunta sobre la razón o la causa de la cantidad de factor que posee ni por qué tiene uno o una combinación de los tres, en otros términos, no le interesa la distribución inicial de factores.

8 Las proposiciones de estos autores y las historias de la corriente utilitarista marginalista se pueden consultar en Dobb (1973), Schumpeter (1954) y Seligman (1962).

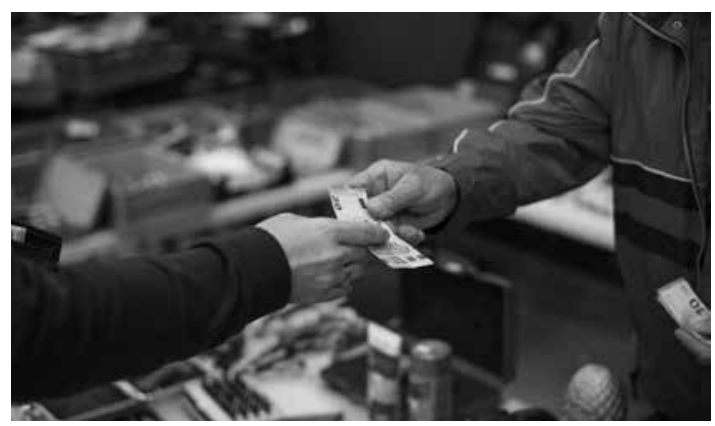

La cantidad de factor que oferta depende de la desutilidad, de la infelicidad que le genera entregar el factor al proceso productivo. En el caso del trabajo, no utilizar el tiempo en ocio (felicidad) y dedicarlo a trabajar (desdicha) lo obliga a valorar en términos del costo de oportunidad entre esas dos posibles elecciones. En este caso el individuo busca tener el mínimo de desutilidad del trabajo y obtener el máximo de felicidad con la cesta que comprará con el ingreso obtenido al vender el trabajo. La cantidad de capital que entrega un individuo también depende de la desutilidad que le genera, del dolor en que incurre al entregarlo a una firma para que lo utilice en la producción. Esta desutilidad también debe ser igual al máximo de utilidad que le genera la cesta que comprará con ese ingreso. Del argumento teórico básico desaparece el factor tierra, el que medio se rescata a finales del siglo XX con la denominada nueva teoría económica urbana.

El precio que se paga a cada factor, esto es, el salario real y el precio real del uso del capital, se determina por la negociación con la firma o empresa. Por el lado del oferente, el individuo, el criterio de decisión es la desutilidad y por el lado del demandante de factores, la firma, es la productividad. El precio finalmente pactado siempre será igual a la productividad del factor. Es un precio de mercado, en el sentido que entre el individuo y la firma, cada uno con su criterio, valora y tranza. El pago es un costo para la firma y un ingreso para el individuo, el cual lo utilizará para comprar la cesta de bienes y servicios preferida. Esa cesta es el ingreso real del individuo. 
De acuerdo con lo anterior, el sistema está montado en términos reales. En el mercado de factores se entrega una cantidad de factor productivo y se recibe a cambio una moneda de intercambio, un vale o cupón o "bancores" (Arrow y Hahn, 1977), que luego se utiliza para comprar la cesta en el mercado de bienes y servicios, para hacer efectivo, el ingreso real individual. Desde luego, la firma es el oferente de bienes y servicios; entonces, al hacer efectivo el ingreso el individuo le oferta los cupones y la empresa los demanda y a cambio le entrega bienes y servicios. Los cupones salieron de la empresa y retornan a la empresa. Estos argumentos se ubican en lo que algunos llaman el espacio de los bienes, sin consideración de las características económicas del dinero, sin referencia a una economía monetaria (Benetti, 1990).

El sujeto central de esta teoría es el individuo, él valora lo que entrega y lo que recibe según los criterios o juicios de valor ya indicados. Valora el costo de oportunidad al entregar factores y valora la felicidad que obtiene al recibir la cesta. En ambos lados del proceso se encuentra un valor relativo. Pero como se manifestó anteriormente, es necesario un valor absoluto. Esta teoría lo obtiene a partir de la felicidad obtenida. El valor que un individuo le da a un bien o servicio es igual a la utilidad que de él deriva. Como lo dice uno de los fundadores: "Añadimos ahora que también la medida del valor es totalmente subjetiva y que, por consiguiente, un bien puede constituir para un sujeto económico un gran valor, para otro un valor menor y para un tercero un valor nulo" (Menger, 1985, p. 131).

Y llegado a este punto entra un aspecto fundamental que aún no se ha señalado. No valora con la cantidad total sino con la última unidad de bien o servicio consumido, porque el consumo de unidades seguidas le genera diferentes niveles de felicidad. La primera unidad le da la mayor felicidad, la última menor utilidad. Esa unidad terminal o marginal es la que, comparada con las anteriores, le genera la mayor desutilidad. Es la famosa ley de la utilidad marginal decreciente. La unidad marginal iguala la utilidad con la desutilidad, la felicidad con el dolor, en que el individuo incurre en su elección de consumo. En consecuencia, el valor de los bienes y servicios quedan medidos en términos de la utilidad de la última unidad, de la unidad marginal. Por eso se dice valoración en términos de la utilidad marginal. Todos los economistas que argumentan con base en la utilidad marginal, aquí los llamo utilitaristas marginalistas. No todo pensador utilitarista es marginalista, y no todo pensador marginalista es utilitarista, todos estos quedan excluidos de la denominación utilitarista marginalista.

Si se valora con arreglo a la utilidad marginal queda una cuestión por resolver: ¿cuál es la unidad de medida de la utilidad marginal? $\mathrm{Al}$ respecto se plantearon dos propuestas de medición. La primera, los utilitaristas marginalistas cardinales propusieron el útil. Si se parte del argumento de que "el valor es de naturaleza subjetiva, no solo en cuanto a su esencia, sino también cuanto a su medida" (Menger, 1985, p. 131), por cuanto un mismo bien es valorado en diferentes cantidades por individuos diferentes, entonces, la medida la construye cada individuo. Hay una medida de un bien por individuo. Cada uno, e independiente de los demás, le asigna un número al bien o servicio de acuerdo con "la importancia de la satisfacción de aquellas necesidades de las que depende nuestra vida" (Menger, 1985, p. 113). El individuo gradúa las necesidades

Cada combinación de bienes y servicios está asociada a un máximo de utilidad o felicidad y cuando hace efectiva su elección el individuo revela

sus preferencias, está dispuesto a pagar un precio por la cesta preferida. 
por orden de importancia y gradúa la felicidad de la cantidad de bien o servicio que satisface la necesidad. El número asignado es el útil. A mayor importancia, mayor el número, la de menor importancia tendrá el número cero. Construye así una escala donde el número "mide" la relación entre el bien o servicio y el valor subjetivo, se imputa un valor individual al bien.

Una cosa es tener una escala y otra es poseer una métrica. La escala dice sobre el orden de felicidad que dan las cantidades, pero no mide el valor de la cantidad del bien. Asignar números no significa medir (recuérdese la cita de Russell en la sección anterior). Pero al limitar la valoración a lo individual el útil no es un estándar universal, no es una magnitud de medida, menos se puede usar para inferir algo sobre lo social. Y no puede ser una magnitud porque no precisa sobre la intensidad del placer que se deriva del consumo, la duración de la felicidad, la intensidad, lo fructífero del placer o su extensión. Si la utilidad de un bien o servicio $\left(u_{i}\right)$ es un número real, no tiene significado alguno decir que la utilidad de la cesta de $n$ bienes y servicios $\left(U_{c}\right)$ es:

$$
U_{c}=\sum_{i=1}^{n} u_{i}
$$

Algunos rechazaron la medición cardinal de la utilidad. "Hemos admitido que esta cosa llamada placer, valor de uso, utilidad económica, ophelimite, era una cantidad, pero la demostración no ha sido dada. Supongamos que se hace esta demostración, ¿cómo se haría para medir esta cantidad?", y "Las nociones de valor de uso, de utilidad de ophelimite, de indicios de ophelimite, etcétera, facilitan mucho la exposición de la teoría del equilibrio económico, pero no son necesarias para construir la teoría" (Pareto 1945, p. 123). Esta opinión implicaría que la economía no necesita una medida cuantitativa de lo que se valora, pese a que todos los agentes económicos tratan con variables que valoran para tomar decisiones. Sin embargo, dio origen a análisis de intercambio que evitaran las magnitudes de utilidad.

Ante las dificultades de la teoría cardinalista (Smart, 1931), a finales de la tercera década del siglo XX se empezaron a plantear alternativas. Hicks y Allen (1934a y 1934b) escribieron el artículo seminal que sentó las bases para abandonar la medición de la utilidad. A partir de los años cuarenta del siglo XX empezó a dominar el planteamiento ordinalista. "If total utility is not quantitatively definable, neither is marginal utility. But the theory of value does not need any precise definition of marginal utility" (Hicks y Allen, 1934a, p. 55). Se abandona la utilidad marginal decreciente. Se trabaja con la relación de dos utilidades marginales. Se creó entonces la tasa marginal de sustitución, que en opinión de Hicks y Allen tiene un significado preciso, siempre y cuando se conozcan las cantidades de las mercancías que tiene el consumidor. Aparece la curva de indiferencia que no requeriría unidad de medida. La utilidad marginal queda en términos del bien en el denominador, más exactamente, la utilidad marginal en el denominador de la relación de sustitución. Hicks (1977) extendió el argumento a la producción, a la teoría de la empresa de manera que los elementos determinantes son la tasa marginal de transformación y la condición de igualdad del producto marginal al precio de factor, y el precio del producto igual al costo marginal. Todo esto sin necesidad de unidad de medida.

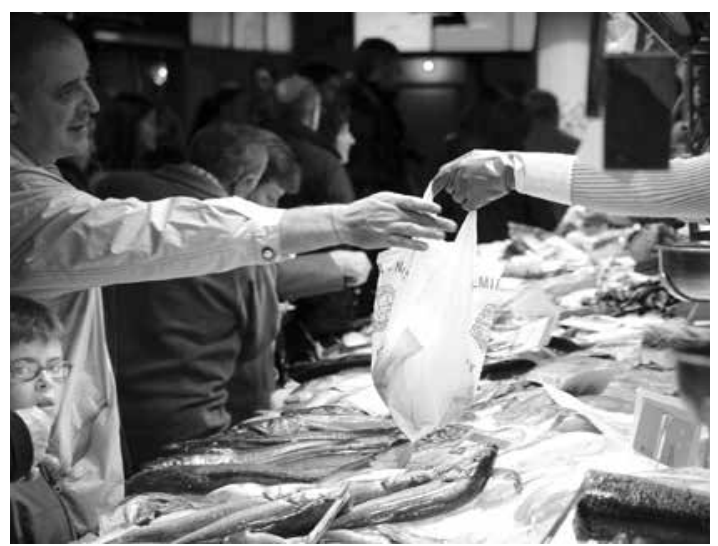


La lógica de preferencias se planteó en términos axiomáticos y se extendió al sistema social, al equilibrio general (Arrow y Hahn, 1977; y Debreu, 1973). En este ámbito, se empieza en el consumidor que puede ser un individuo o una familia (Debreu, 1973), y se pasa a un vector de familias; se plantea un productor y se pasa al conjunto de la producción. Sin embargo, la parte activa, determinante, es el consumidor. Su elección presenta continuidad sobre las preferencias y esto permite asignarle un número a la clase de indiferencia, donde el número mayor indica el más preferido. Entonces, el conjunto que está plenamente preordenado por las preferencias contiene una función de valor real creciente que se llama función de utilidad; de manera que "dado un preorden de preferencias, una función de utilidad que le corresponda es en gran medida arbitraria" (Debreu, 1973, p. 72). La parte pasiva, la empresa, se limita a elegir de un conjunto factible el nivel de producto, su oferta al mercado, de manera que maximice su beneficio, cuando el beneficio marginal es cero.

En la versión ordinalista se obtienen funciones de indiferencia. Unas superiores o inferiores a una función dada. ¿Cómo se mide la distancia entre dos funciones? No hay unidad de medida, tan solo se conoce que una es superior o inferior a la de referencia.

El utilitarismo marginalista mide cantidades relativas de bienes y servicios que le permite determinar la cantidad demandada y ofertada de un bien o de un conjunto de bienes y servicios, pero siempre y cuando el precio lo determine el mercado. ¿Cómo se mide ese precio? "A cada mercancía, digamos la $h$-ésima, se asocia un número real, su precio, $p_{h}$ " (Debreu, 1973, p. 42). Ese número equivale, tanto en equilibrio parcial como en equilibrio general, a un bien, del bien numerario. En el fondo es el retorno, por un camino elegante, a la idea de Ricardo de un bien que sirve para determinar el valor relativo, el oro, que en este caso se llamará el bancores. Y si se

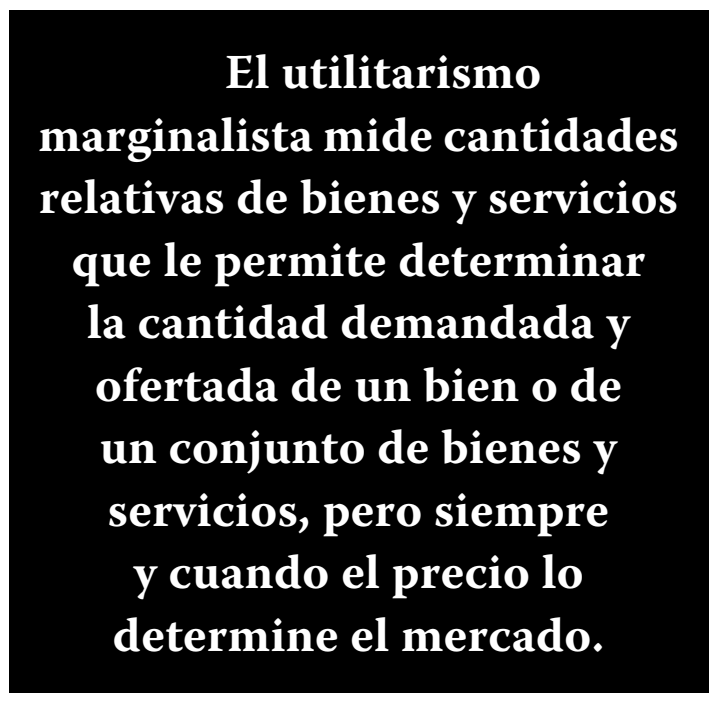

debe introducir el dinero, ¿cómo se determina el valor del dinero?

La inconformidad con el utilitarismo marginalista y las sin salidas de la teoría de los precios de producción llevaron a escepticismo frente a la necesidad de una teoría del valor para construir un marco teórico general, como lo dijo la profesora Robinson, y lo sostiene la escuela de pensamiento poskeynesiana; $\mathrm{o}$ a trabajar a nivel agregado con medidas parciales o no integradas como lo hace Keynes (1986) para afrontar "Lo poco satisfactorio de las unidades que emplean los economistas puede ejemplificarse por los conceptos de dividendo nacional, existencia (o acervo) de capital real y nivel general de precios" (p. 43); o a concentrarse en precios monetarios sin hacer referencia a precios de producción y a funciones de utilidad; o a replantear todo el sistema de ecuaciones o sistema económico con base en un planteamiento monetario o con las teorías del circuito monetario o a replantear la relación entre el precio y el valor (Benetti, 1990; Cencini, 1984; Elder-Vass, 2019; Graziani, 2003; Matallana, 2016); o continuar un legado de análisis integral como el que dejaron Sraffa (1960), Garegnani (1970, 1982, 1984) y Pasinetti (1962, 1978), donde se pueda determinar la medición del capital, la tasa de ganancia y la tasa de salarios (teoría de la distribución). 


\section{Conclusión}

Si el análisis económico trata de esclarecer las relaciones entre aspectos cuantitativos fundamentales de la sociedad, la teoría del valor es la piedra angular para alcanzar un cuerpo analítico con hipótesis coherentes y consistentes que permita interpretar la dinámica del sistema económico de mercado. La cuestión de la medida del valor sigue abierta, tanto en lo que corresponde a su unidad de medida como en su relación con las teorías de la producción, distribución, precios y monetaria. Al no poseer una unidad de medida, cuestiones teóricas y operativas se evaporan y dejan una grieta conceptual, que se debe cerrar, pues las consecuencias prácticas no son de poca importancia. Sin medida, muchas de las conclusiones de los modelos de política monetaria, cambiaria, fiscal, sectorial, crecimiento económico, entre otros, quedan seriamente en entredicho.

\section{Referencias}

Arrow, K. J. y Hahn, F. H. (1977). Análisis general competitivo. E. L. Suárez (Trad.). México D.F.: Fondo de Cultura Económica.

Benetti, C. (1990). Moneda y teoría del valor. México D. F.: Fondo de Cultura Económica. Benetti, C. (1983). Dinero, precios y equilibrio. Lecturas de Economía, 12.

Boumans, M. (Editor [2007]). Measurement in Economics, A Handbook. New York: Elsevier.

Boumans, M. (2015). Science Outside the Laboratory: Measurement in Field Science and Economics. New York: Oxford University.

Cantillon, R. (1950). Ensayo sobre la naturaleza del comercio en general. M. Sánchez S. (Trad.). México D. F.: Fondo de Cultura Económica.

Cataño, J. F. (2009). Lecciones de economía marxista: Mercados, precios y dinero desde un enfoque heterodoxo. Bogotá D. C.: Universidad Nacional de Colombia.

Cencini, A. (1984). Time and the macroeconomic analysis of income. New York: St. Martin.

Cuevas, H. (1986). Valor y sistema de precios. Bogotá D. E.: Universidad Nacional de Colombia.

Debreu, G. (1973). Teoría del valor, Un análisis axiomático del equilibrio económico. A. Mas Colell y J. Oliu (Trad.). Barcelona: Bosch.
Dobb, M. (1945). Economía política y capitalismo. E. Martínez (Trad.). México D. F.: Fondo de Cultura Económica.

Dobb, M. (1978). Teorías del valor y de la distribución desde Adam Smith, ideología y teoría económica. R. Cusminsky (Trad.). México D. F. Siglo veintiuno.

Dostaler, G. (1980). Valor y precio, Historia de un debate. F. Angulo (Trad.). México D. F.: Terra Nova.

Elder-Vass, D. (2019). No Price Without Value: Towards a Theory of Value and Price. Cambridge Journal of Economics, 43(6), 1485-1498.

Garegnani, P. (1982). El capital en la teoría de la distribución. J. A. García (Trad.). Barcelona: Oikos-Tau.

Garegnani, P. (1970). Heterogeneous Capital, the Production Function and the Theory of Distribution. The Review of Economic Studies, 37(3), 407-436.

Garegnani, P. (1984). Value and distribution in the classical economist and Marx. Oxford Economic Papers, 36(2), pp. 291-325.

Graziani, A. (2003). The Monetary Circuit of Production. Cambridge: Cambridge Universtity Press.

Haavelmo, T. (1941). On the Theory and Measurement of Economic Relations. Cambridge-Massachusetts: Mimeograph. Recuperado de https://www.sv.uio.no/econ/en 
glish/research/networks/haavelmo-network/publications/files/TH1941c.pdf

Hicks, J. R. y Allen, R. G. D. (1934a). A Reconsideration of the Theory of Value Part I. Economica, New Series, 1(1), pp. 52-76

Hicks, J. R. y Allen, R. G. D. (1934b). A Reconsideration of the Theory of Value Part II, A mathematical Theory of Individual Demand Functions. Economica, New Series, 1(2), 196-219.

Hicks, J. R. (1977). Valor y capital. J. Márquez (Trad.). México D. F.: Fondo de Cultura Económica.

Hilferding, R., Von Böhm-Bawerk, E. y Von Bortkiewicz, L. (1974). Economía burguesa y economía socialista. C. Manzoni (Trad.). Córdoba: Cuadernos de Pasado y Presente núm. 49.

Hollander, S. (1988). La economía de David Ricardo. J. Fernández (Trad.). México D. F.: Fondo de Cultura Económica.

Hunt, E. K. y Schwartz, J. G. (1977). Crítica de la teoría económica. E. L. Suárez (Trad.), México D. F.: Fondo de Cultura Económica.

Keynes, J. M., (1986). Teoría general de la ocupación, el interés y el dinero. E. Hornedo (Trad.). México D. F.: Fondo de Cultura Económica.

MacVane, S. M. (1893). The Austrian Theory of Value. The Annals of the American Academy of Political and Social Science, 4, 12-41.

Matallana, H. (2016). Dinero endógeno, circuito monetario y estado estacionario en la economía monetaria de producción Ensayos de Economía, 26(49), 47-87.

Meek, R. L. (1956). Studies in the Labor Theory. New York: Monthly Review.

Marx, K. (2001). El capital. Crítica de la economía política. Tomo I, Vol. 1. P. Scaron (Trad.). México D. F.: Siglo veintiuno.

Menger, C. (1985). Principios de economía política. M. Villanueva (Trad.). Barcelona: Orbis.

Michell, J. (2007). Representational Theory of Measurement.
Morgan, M. S. (2007). An Analytical History of Measuring Practices, The Case of Velocities of Money.

Pareto, V. (1945). Manual de Economía Política. G. Casanellas (Trad.). Buenos Aires: Atalaya.

Pasinetti, L. L. (1962). Rate of Profit and Income Distribution in Relation to the Rate of Economic Growth. The Review of Economic Studies, 29(4), 267-279.

Pasinetti, L. L. (1978). Wicksell Effects and Reswitchings of Technique in Capital Theory. The Scandinavian Journal of Economics, 80(2), 181-189.

Petty, W. (1899). A Treatise of Taxes $\mathcal{E}$ Contributions. London: N. Brooke.

Petty, W. (1899). Political Arithmetic, or A Discourse Concerning. London: Robert Clavel at et Peacock.

Petty, W. (1899). The Economic Writings of Sir William Petty, together with The Observations upon Bills of Mortality, more probably by Captian John Graunt, Vol. 1. C. H. Hull (Edit.). London: Cambridge University Press. Recuperado de https://oll. libertyfund.org/titles/petty-the-economicwritings-of-sir-william-petty-2-vols

Ricardo, D. (1960). Valor absoluto y valor en cambio, en Obras y correspondencia, Vol. $I V$. F. M. Torner (Trad.). México D. F.: Fondo de Cultura Económica.

Ricardo, D. (1973). Principios de economía política y tributación. J. Broc, N. Wolff y J. Estrada (Trad.). México D. F.: Fondo de Cultura Económica.

Russell, B. (1983). El conocimiento humano. N. Míguez (Trad.). Barcelona: Orbis.

Schumpeter, J. A. (1982). Historia del análisis económico. M. Sacristán (Trad.). Barcelona: Ariel.

Seligman, B. B. (1967). Principales corrientes de la ciencia económica moderna, el pensamiento económico después de 1870. A. Casahuga, J. A. García-Durán, P. Maragall y F. Rahola (Trad.). Barcelona: Oikos-tau.

Shackle, G. L. S. (1976). Epistémica y economía, Crítica de las doctrinas económicas. F. 
González (Trad.). México D. F. Fondo de Cultura Económica.

Sherman, H. J. (1972). El valor y la asignación del mercado.

Smart, W. (1931). An Introduction to the Theory of Value on the Linews of Menger, Wieser, and Böhm-Bawerk. London: MacMillan.

Smith, A. (1981). Investigación sobre la naturaleza y causas de la riqueza de las naciones. G. Franco (Trad.). México D. F.: Fondo de Cultura Económica.

Sraffa, P. (1965). Producción de mercancías por medio de mercancías. L. A. Rojo (Trad.). Barcelona: Oikos-Tau.
Steedman, I. (1985). Marx, Sraffa y el problema de la transformación. E. L. Suárez (Trad.). México D. F.: Fondo de Cultura Económica.

Tsoulfidis L. y P. Tsaliki (2019). Classical Political Economics and Modern Capitalism, Theories of Value, Competition, Trade and Long Cycles. Suiza: Springer.

Wright, I. (2019). Marx's transformation problem and Pasinetti's vertically integrated subsystems. Cambridge Journal of Economics, 43(1) 169-186. 